Controversial glycosaminoglycan conformations

SIR-Despite major recent advances in the conformational analysis of glycosaminoglycans, several controversies remain concerning the interpretation of physical and chemical measurements. One major issue concerns the ring form of $\mathrm{L}$ iduronate residues in a number of important glycans and proteoglycans having dermatan, heparin or heparan chains. At least for dermatan sulphate, the evidence from diffraction analysis ${ }^{1}$ is that these residues in crystalline fibres are close to the form having the carboxylate group axial $\left({ }^{+} \mathrm{C}_{1}\right.$, Fig. $1)$. In solution, however, this would not preclude the alternative chair ( ${ }^{\prime} \mathrm{C}_{\downarrow}$, Fig. 1) since it is well known that the interaction energies are such that equilibrium between the two forms is delicately balanced ${ }^{2}$ and there is of course no reason a priori why the crystalline form should persist into solution. Also possible are various distorted chairs and skew boats but these are not discussed here since their consideration would constitute a second order of analysis.

Two conflicting arguments have been put forward. On the one hand, the small proton nuclear magnetic resonance vicinal coupling constrants around the ring would point ${ }^{3}$ to ${ }^{\prime} \mathrm{C}_{3}$, an assignment that is confirmed by our recent studies by circular dichroism ${ }^{4}$. On the other hand ${ }^{5.6}$, the extreme susceptibility of these residues to periodate oxidation must be taken as a demonstration of an ability to form a cyclic reaction intermediate which requires a small dihedral angle between the $0(2)$ and 0 (3) substituents, as in the ${ }^{4} \mathrm{C}_{1}$ (Fig. 1). We now write to point out that this apparent discrepancy can be resolved by the application of established stereochemical principles which show that the periodate

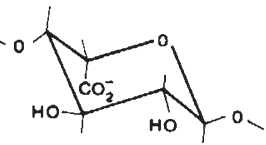

${ }^{4} \mathrm{C}$,

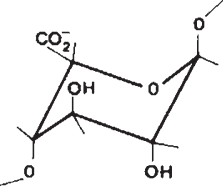

'C.
Fig. 1 Alternative chair conformations for $\mathrm{L}$ iduronate, with designations as used in text.

oxidation behaviour can be understood without the need to postulate a preponderance of ${ }^{4} \mathrm{C}_{1}$ in the equilibrium between ground state conformations.

It follows from the Curtin-Hammett principle that - when alternative conformers of a reactive compound are in rapid equilibrium relative to the rates of their subsequent reaction - the reaction pathway is independent of their relative ground state energy levels (abundancies); thus a major conformer (in this case ${ }^{1} \mathrm{C}_{4}$ ) can function as an unreactive reservoir species while a very minor conformer $\left({ }^{4} \mathrm{C}_{1}\right)$ determines the course of reaction. Another very striking example of this principle has been described recently dur- ing the catalytic asymmetric hydrogenation of a prochiral dehydro-amino acid where the reactive intermediate of a rapidly equilibrating pair was actually undetectable. This leads to the postulate that the iduronate and glucuronate residues are oxidized in similar conformations, in which case one might at first expect the latter to react more quickly because of the greater probability of productive collisions - whereas the reverse might be the case. This problem can be resolved by reference to a kinetic study of the cyclohexane-1,2-diols which provides evidence that the release of crowding in their cyclic intermediates in periodate oxidation can provide the driving force for steric acceleration, at least at $p \mathrm{H} 9$ (ref. 9). We suggest that this indicates that transition states of this type are sufficiently productlike in character (that is, C-C bond cleavage is well progressed) for steric crowding to be substantially relieved. The energy levels for the transition states for the glucuronate and iduronate pathways would then be closer in energy than those for their ground state conformations. The higher ground state energy of iduronate relative to that of glucuronate - as a result of the many unfavourable internal interactions - would therefore provide steric acceleration and hence explain the fast rate that is observed for iduronate.

D.A. REES

National Institute for Medical Research,

The Ridgeway, Mill Hill,

London NW7 IAA, UK

Department of Food Research

E.R. MORRIS and Technology,

Cranfield Institute of Technology,

Silsoe College, Silsoe,

Bedford MK45 4DT, UK

Department of Chemistry

J.F. STODDART

University of Sheffield.

Sheffield S3 7HF, UK

E.S.Stevens

Department of Che mistry

State University of New York,

Binghamton, New York 13901, USA

1. Mitra. A.K.. Arnott. S., Isaac, D.H.\& Atkins. E.D.T. J. molec. Biol. 169, 873-901 (1983)

2. Eliel, E.L., Allinger. N.L.. Angyal, S.J. \& Morrison. G.A. Conformational Analysis, 370) (Wiley. New York, 1965).

3. Perlin. A.S. Casu. B.. Sanderson. G.R. \& Tse. J. Carbohy drate Res. 21, 123-132 (1972)

. Csiner, D.G.. Stevens, E.S., Morris. E.R. \& Rees, D.A.J Am. chem. Soc. (in the press)

Scott. J.E. \& Tigwell. M.J. Biochem. J. 173, 103-114 (1978)

Fransson, L.-Å. Carbohydrate Res. 62. 235-244 (1978).

7. Eliel. E.L. Stereochemistry of Carbon Compounds $151-156$ (McGraw Hill. New York. 1962).

8. Halpern, J. Pure appl. Chem. 55, 99-106 (1983)

9. Buist. G.J., Bunton, C. A. \& Miles. J.H. J. C. Soc., 743-748 (1959)

\section{Human lymphocytopathic retroviruses (HLRV)?}

SIR-Owing to the independent discovery of human lymphocytopathic viruses (HLV), called human T-cell leukaemia viruses (HTLV-I,II,III) and, later, human T-cell lymphotropic viruses by Gallo and colleagues at the US National Institute of Health $^{1.2}$; lymphadenopathy-associated viruses (LAV) by Montagnier and colleagues at the Institut Pasteur in Paris; or AIDS-associated retroviruses (ARV) by Levy and associates in San Francisco ${ }^{3}$ there has been lively discussion ${ }^{1.3}$ with respect to naming the heterogenomic retroviridae causing analogous spectra of devastating diseases in humans, cats, monkeys, cattle and mice. Conforming with nomenclature in the other mammals and considering heterogeneity, as well as multiplicity, HLV (in the plural) might constitute a comprehensive abbreviation to connote the leukaemogenic, lymphotropic, lymphocytotropic, lymphocytostatic, lymphocytotoxic, sometimes lymphocytolytic nature of such insidious retroviruses accustomed to use human lymphocytes for reproduction, as well as transport through lymph, blood and other secretions $^{4.5}$

However, HLRV might prove to be a better abbreviation for human lymphocytopathic retroviruses because:

(1) $\mathrm{H}$, standing for human, can be replaced with $\mathrm{Fe}, \mathrm{S}, \mathrm{B}, \mathrm{Mu}$ or any other suitable symbols to designate other creatures ranging from aardvarks to zebras infected with similar retroviridae causing analogous diseases.

(2) T- might be misleading, since lymphocytopathic retroviridae may infect " $B$ cells", as well as other cells in sundry species. So, after Lord Admiral Horatio Nelson at Trafalgar, one might X the "T-". (3) $\mathrm{L}$ related to lymphocytopathies, whether the retroviral effects are leukaemic, lymphomatous, lymphoproliferative, lymphocytostatic, lymphocytolytic, lymphocytopoenic or otherwise lymphocytotropic.

(4) A for AIDS is not comprehensive, since the lymphocytopathic retroviridae may alternatively or simultaneously cause GLS or lymphomas. As an abbreviation for associated, $A$ is superfluous. So, one might $X$ the " $A$ " similarly.

(5) $\mathrm{R}$ for retro denotes DNA retrotranscription by retroviridae which lack double helices and, thus, depend for replication on cells with relatively great DNA concentrations, especially lymphocytes.

(6) $\mathrm{V}$ for viruses must stand, especially if preceded by retro-

(7) While HTLARV sequentially connotes the works of Drs. Gallo, Montagnier and Levy, the abbreviation is a bit awkward. After the K (Köchèl) subclassified works of Wolfgang Amadeus Mozart, it might prove efficient to suffix the HLRV with Arabic numbers $1,2,3$, et cetera, ad infinitum (especially because it appears now that too many different antigenic strains will be found to use roman numerals conveniently). JACK W. SHIELdS Santa Barbara Medical Foundation Clinic, PO Box 1200, Santa Barbara,

California 93102, USA

1. Wong-Staal, F. \& Gallo, R.C. Blood 65,253-263 (1985)

2. Wong-Staal, F. Nature 314, 574 (1985).

. Wong-Staal, F. Nature 314, S74 (1985).

MarX. J.L. Science 227, 1449-1451 (1985).
Shields. J.W. Blood 62 (Suppl 1). 117 a (1983).

4. Shields. J.W. Blood 62 (Suppl 1). 117a (1983).
5. Olsen G.P. \& Shields J.W. Nature 309, 116-117 (1984) 